die Darstellungen von sexuellem Missbrauch und sexueller Gewalt in den Medien von Stereotypien und Fehlinformationen in Bezug auf die Täter- und Opferbilder und auf die Ursachen und Folgen gekennzeichnet sind. Dies wiederum hat zur Konsequenz, dass ein sachgerechter Umgang mit der Problematik erschwert wird und hier Journalisten entsprechend gefordert sind, sensibler und genauer mit diesem Thema umzugehen. Nur so wird es wahrscheinlich, dass sich ein realitätsnahes Problembewusstsein der Rezipienten entwikkeln kann und eine Handlungsbereitschaft im Falle einer Konfrontation mit dem Problem gegeben ist. Es verbleibt zu hoffen, dass das Buch seinen Platz auf den Leselisten insbesondere für die Disziplinen „Medienethik“ und „Qualitätsjournalismus" finden wird.

Dagmar Hoffmann

\section{Norbert Baumgärtner}

\section{Risiko- und Krisenkommunikation}

Rahmenbedingungen, Herausforderungen und Erfolgsfaktoren, dargestellt am Beispiel der chemischen Industrie

München: Verlag Dr. Hut, 2005. - 436 S.

ISBN 3-89963-172-2

Mit seiner Dissertation legt Norbert Baumgärtner eine anwendungsorientierte Forschungsarbeit vor, die das Feld der Unternehmenskommunikation in Risiko- und Krisensituationen systematisch beleuchtet. Seine Arbeit umfasst sowohl eine Begriffsbestimmung und theoretische Herleitung aus verschiedenen Disziplinen als auch eine empirische Umsetzung.

Nach einer problematisierenden Bestimmung seiner zentralen Untersuchungsgegenstände Risiko und Krise in Abgrenzung zu angrenzenden Begriffen wie Konflikt oder Katastrophe, deren Verwendung in der Praxis häufig schwammig ist, stellt Baumgärtner angenehm kurz den jeweils aktuellen Forschungsstand zu Risiko- und Krisenkommunikation dar und erörtert defizitanalytisch deren praktische Relevanz. Bereits hier wird deutlich, dass die öffentliche Debatte von Risiken unterschiedliche Fragen im Hinblick auf deren Objektivierbarkeit insbesondere aus Experten- und Laienperspektive aufwirft, da in diesen Gruppen mit unterschiedlichen Risikokonstruktionen operiert wird: Die Messverfahren bzw. Einflussfaktoren der Risikowahrnehmung der Experten basiert quantifizierend auf einem naturwissenschaftlich-technischen, statistischen Vorgehen, während Laien vereinfachende mentale Heuristiken anwenden und eine dementsprechend vorwiegend qualitative, vage und offene Risikovorstellung haben, deren Gebrauch innerhalb der Gruppe uneinheitlich ist.

Risikokommunikation muss daher immer beide Perspektiven berücksichtigen. Ihre Hauptfunktionen fasst Baumgärtner in Anlehnung an Keeney/von Winterfeldt, Renn/Levine, Renn/Kastenholz und Hribal als Befriedigung des ,Rechts auf Wissen' (S. 158) zusammen. Damit ist die Erfüllung der von staatlicher Seite gesetzten Normen hinsichtlich der Informationspflicht an die Öffentlichkeit genauso gemeint wie eine ganze Reihe weiter gehender Maßnahmen: die Aufklärung der Öffentlichkeit über Risiken, eine Wissensverbesserung, die zielorientierte Veränderung von Einstellungen der Öffentlichkeit, eine Verhaltensänderung beim Verursacher und beim Rezipienten, die Legitimation von Zielen, die Einbeziehung der Betroffenen ins Risikomanagement, die Information über geeignete Maßnahmen zur Risikoreduzierung bzw. -minimierung, die Vorbereitung auf mögliche Notfälle als handlungsunterstützende Information, die Verbesserung des Verständnisses von Werten und Besorgnissen der Öffentlichkeit bei den Experten, eine beidseitige Erhöhung des Vertrauens und der Glaubwürdigkeit, die Vermeidung von Konflikteskalationen sowie schlussendlich eine Konfliktlösung. Damit Risikokommunikation diese Funktionen wahrnehmen kann, bedarf es der eingehenden Analyse des gesellschaftspolitischen Umfelds, in dem öffentliche Konflikte ausgetragen werden. In seinem Grundlagenband skizziert Baumgärtner mit Hilfe des Arena-Modells (v. a. nach Renn) diesen öffentlichen Aktionsraum und analysiert die in der Arena tätigen Akteure hinsichtlich der konstitutiven Mechanismen zur Bildung von Anspruchsgruppen, ihrer Sanktionsmacht, ihren Legitimations- und Interaktionsmöglichkeiten. Baumgärtner arbeitet in einem weiteren Arbeitsschritt prägnant die unterschiedlichen Erwartungshaltungen der Akteure an das Mediensystem heraus, dem eine entscheidende Vermittlerrolle in der Risiko- und Krisenkommunikation zukommt. Hierzu zieht er kommunikationswissenschaftliche Theorien und Modelle, wie Stimulus-Response, Two-Step- 
Flow of Communication, Agenda-Setting, Uses-and-Gratifications und Schweigespirale, heran. Er kommt zu dem wenig überraschenden Schluss, dass „so wenig die Kommunikationswissenschaft zu einem definitiven Urteil“ kommen könne, „unter welchen Umständen welche Medien welche (und wie starke) Wirkungen haben, so wenig [...] das dieses Buch leisten" könne.

Anhand von Fallstudien zu sechs Krisen von Chemieunternehmen zeigt Baumgärtner auf, dass es unabhängig von den großen Theorieund Modellkonstrukten der Kommunikationswissenschaft generalisierte Erfolgsfaktoren für die Unternehmenskommunikation gibt, die sich mit den im Theorieteil seiner Arbeit angestellten Überlegungen begründen lassen.

An einigen Stellen ist auffällig, dass der Autor etwas zu Zitatenreihungen neigt. Darüber hinaus bleibt die Studie leider in einigen Bereichen, wie z. B. den Lebenszyklen von Risikothemen, an der Oberfläche. Damit soll aber nicht in oftmals review-typisches Kritisieren von Beschränkungen wissenschaftlicher Arbeiten auf ein Gebiet verfallen werden: Der Autor liefert mit diesem Grundlagenwerk einen wichtigen, praxisorientierten Beitrag zum Verständnis der Risiko- und Krisenkommunikation, der erfolgreich den Spagat zwischen wissenschaftlicher Kontextualisierung und anwendungsorientiertem Fokus meistert.

Steffen Kolb \& Steffen Burkhardt

\section{Jens Damm/Simona Thomas (Hrsg.)}

\section{Chinese Cyberspaces}

Technological Changes and Political Effects

London/New York: Routledge, 2006. - 180 S.

ISBN10: 0-415-33208-7

ISBN13: 9-78-0-415-33208-8

"Policy follows technology" - diese Gesetzmäßigkeit gilt auch für den durch beständiges Wachstum gekennzeichneten chinesischen Markt der audiovisuellen, elektronischen Medien. Ist die ordnungspolitische Ausgestaltung des chinesischen Fernsehmarktes durchaus mit den bundesdeutschen Strukturen vergleichbar? Halt! Natürlich nur im Vergleich zum systematischen Aufbau zuständiger, regulierender Institutionen und natürlich (leider?, noch?, kulturell?) nicht aus der Perspektive des herrschenden politischen Paradigmas, respektive verfassungsgemäßen Umsetzungen im Sinne der gesamten Gesellschaft.

Ganz und gar nicht vergleichbar stellt sich indes der administrative Umgang mit virtuellen Welten und dem Internet als Ganzem in China dar. Der von Jens Damm und Simona Thomas herausgegebene und überwiegend sekundäranalytische Sammelband Chinese Cyberspaces bietet laut Klappentext eine multidisziplinäre Auseinandersetzung um die Entwicklungen des Internets in China aus sozialwissenschaftlicher, politischer, ökonomischer und umgreifend aus kultureller Perspektive mit den daraus folgenden Konsequenzen: Der Etablierung von Aufsichtsbehörden zur Sicherstellung der politischen Stabilität im Feld der Individual- und Massenkommunikation im Cyberspace.

Der Band untergliedert sich in sieben Beiträge und beginnt mit einer Einleitung der Herausgeber zur Rolle des Internets in China (sowie einem Überblick zum aktuellen Forschungsstand), die, begleitet durch den fortschreitenden Öffnungsprozess der Volksrepublik, sich seit dem Ende der neunziger Jahre sowohl gesellschaftlich als auch ökonomisch als Teil der Lebensrealität - zumindest in den Ballungszentren - herausgebildet hat, wenngleich unter restriktiver Regulierung des Zugangs und der Art der über dieses Medium verbreiteten Inhalte. Der die Einführung schließende Verweis auf den Kontrollanspruch der Staatsführung leitet gleichermaßen auf den zweiten Beitrag von Eric Harwit und Duncan Clark über. Schon zu Beginn des Bandes wird eines besonders deutlich: Das Internet in China ist nicht mit dem beispielsweise ,europäischen' Internet vergleichbar und kann vor diesem Hintergrund vertretbar und in Folge als CWW (Chinese Wide Web) und nicht als WWW klassifiziert werden. Die Autoren zeichnen die infrastrukturelle Entwicklung nach, die mit heutigem Stand übergreifend durch das MII (Ministry of Information Industry) bestimmt ist. Ähneln sich die soziodemographischen Daten und genutzte Funktionen wie E-Mail oder Unterhaltungsangebote im hier gezogenen Vergleich mit den USA sehr, so fällt doch auf, dass insbesondere Reiseinformationen (gering) und die Teilnahme an Chatrooms (hoch) divergieren. Diese als Folge der politischen Prämissen zur Regulierung des Internets zu interpretierende Bedeutung einzelner Kommunikationsräume steht in direktem Zusammenhang mit einer absurd erscheinenden Policy: der nahezu 